\title{
An asymmetry detected in the disk of $\kappa$ Canis Majoris with AMBER/VLTI*
}

A. Meilland ${ }^{1}$, F. Millour 2,3 , P. Stee ${ }^{1}$, A. Domiciano de Souza ${ }^{2,1}$, R.G. Petrov², D. Mourard ${ }^{1}$, S. Jankov $^{2}$, S. Robbe-Dubois ${ }^{2}$, A. Spang ${ }^{1}$, E. Aristidi ${ }^{2}$, P. Antonelli ${ }^{1}$, U. Beckmann ${ }^{4}$, Y. Bresson ${ }^{1}$, A. Chelli $^{3}$, M. Dugué $^{1}$, G. Duvert ${ }^{3}$, S. Gennari ${ }^{5}$, L. Glück ${ }^{3}$, P. Kern ${ }^{3}$, S. Lagarde ${ }^{1}$, E. Le Coarer ${ }^{3}$, F. Lisi ${ }^{5}$, F. Malbet ${ }^{3}$, K. Perraut ${ }^{3}$, P. Puget $^{3}$,

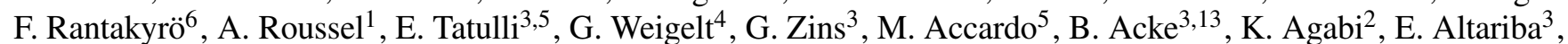
B. Arezki ${ }^{3}$, C. Baffa ${ }^{5}$, J. Behrend ${ }^{4}$, T. Blöcker ${ }^{4}$, S. Bonhomme ${ }^{1}$, S. Busoni ${ }^{5}$, F. Cassaing ${ }^{7}$, J.-M. Clausse $^{1}$, J. Colin $^{1}$, C. Connot ${ }^{4}$, A. Delboulbé ${ }^{3}$, T. Driebe ${ }^{4}$, P. Feautrier ${ }^{3}$, D. Ferruzzi ${ }^{5}$, T. Forveille ${ }^{3}$, E. Fossat ${ }^{2}$, R. Foy $^{8}$, D. Fraix-Burnet $^{3}$, A. Gallardo ${ }^{3}$, E. Giani ${ }^{5}$, C. Gil ${ }^{3,14}$, A. Glentzlin ${ }^{1}$, M. Heiden ${ }^{4}$, M. Heininger ${ }^{4}$, O. Hernandez Utrera ${ }^{3}$, K.-H. Hofmann ${ }^{4}$, D. Kamm ${ }^{1}$, M. Kiekebusch ${ }^{6}$, S. Kraus ${ }^{4}$, D. Le Contel ${ }^{1}$, J.-M. Le Contel ${ }^{1}$, T. Lesourd ${ }^{9}$, B. Lopez ${ }^{1}$, M. Lopez ${ }^{9}$, Y. Magnard $^{3}$, A. Marconi ${ }^{5}$, G. Mars ${ }^{1}$, G. Martinot-Lagarde ${ }^{9,1}$, P. Mathias ${ }^{1}$, P. Mège ${ }^{3}$, J.-L. Monin ${ }^{3}$, D. Mouillet ${ }^{3,15}$, E. Nussbaum ${ }^{4}$, K. Ohnaka ${ }^{4}$, J. Pacheco ${ }^{1}$, C. Perrier ${ }^{3}$, Y. Rabbia ${ }^{1}$, S. Rebattu ${ }^{1}$, F. Reynaud ${ }^{10}$, A. Richichi ${ }^{11}$, A. Robini ${ }^{2}$, M. Sacchettini ${ }^{3}$, D. Schertl ${ }^{4}$, M. Schöller ${ }^{6}$, W. Solscheid ${ }^{4}$, P. Stefanini ${ }^{5}$, M. Tallon ${ }^{8}$, I. Tallon-Bosc ${ }^{8}$, D. Tasso ${ }^{1}$, L. Testi ${ }^{5}$, F. Vakili ${ }^{2}$, O. von der Lühe ${ }^{12}$, J.-C. Valtier $^{1}$, M. Vannier ${ }^{2,6,16}$, and N. Ventura ${ }^{3}$

(Affiliations can be found after the references)

Received 11 April 2006 / Accepted 25 October 2006

\section{ABSTRACT}

\begin{abstract}
Aims. We study the geometry and kinematics of the circumstellar environment of the $\mathrm{Be}$ star $\kappa \mathrm{CMa}$ in the $\mathrm{Br} \gamma$ emission line and its nearby continuum.

Methods. We use the AMBER/VLTI instrument operating in the $K$ band, which provides a spatial resolution of about 6 mas with a spectral resolution of 1500 , to study the kinematics within the disk and to infer its rotation law. To obtain more kinematical constraints we also use a high spectral resolution $\mathrm{Pa} \beta$ line profile obtain in December 2005 at the Observatorio do Pico do Dios, Brazil and we compile $V / R$ line profile variations and spectral energy distribution data points from the literature.

Results. Using differential visibilities and differential phases across the $\operatorname{Br} \gamma$ line we detect an asymmetry in the disk. Moreover, we found that $\kappa$ CMa seems difficult to fit within the classical scenario for Be stars, illustrated recently by $\alpha$ Arae observations, i.e. a fast rotating B star close to its breakup velocity surrounded by a Keplerian circumstellar disk with an enhanced polar wind. We discuss the possibility that $\kappa$ CMa is a critical rotator with a Keplerian rotating disk and examine whether if the detected asymmetry can be interpreted within the "one-armed" viscous disk framework.
\end{abstract}

Key words. techniques: high angular resolution - techniques: interferometric - stars: emission-line, Be - stars: individual: Keplerian rotation stars: individual: $\kappa \mathrm{CMa}-$ stars: circumstellar matter

\section{Introduction}

The "Be phenomenon" is related to hot stars that have exhibited Balmer lines at least once in emission, with infrared excess produced by free-free and free-bound processes in an extended circumstellar disk. There is now strong evidence that the disk around the Be star $\alpha$ Arae is Keplerian (Meilland et al. 2007) and that this dense equatorial disk is slowly expanding. However, there is also evidence for a polar enhanced wind. This was predicted for almost critically rotating stars, and thus for a large fraction of Be stars. Recently, Kervella \& Domiciano de Souza (2006) showed an enhanced polar wind for the Be star Achernar ever though this Be star presents no hydrogen lines in strong emission. Thus, it seems that a significant polar wind may be present even if the star is still in a normal B phase, signifying that this enhanced polar wind is not related to a dense equatorial envelope. However many issues remain unsolved about the structure of the circumstellar

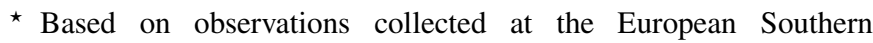
Observatory, Paranal, Chile, within the guaranteed time programme 074.A-9025(A). envelope in Be stars, which probably depends on the dominant mass ejection mechanisms from the central star and on the way the ejected mass is redistributed in the near circumstellar environment. Meilland et al. (2006) reported theoretical spectral energy distributions (SEDs), Br $\gamma$ line profiles and visibilities for two likely scenarios of the disk dissipation of active hot stars, and account for the transition from the Be to the B spectroscopic phase.

$\kappa$ CMa (HD 50013, HR 2538) is one the brightest Be stars in the southern hemisphere $(V=3.8, K=3.6)$. It is classified as a B2IVe star, and the distance deduced from the Hipparcos parallax is $230 \pm 30 \mathrm{pc}$. The measured $v \sin i$ values range from $220 \mathrm{~km} \mathrm{~s}^{-1}$ (Dachs et al. 1989; Mennickent et al. 2004; Okazaki 1997; Prinja 1989) to $243 \mathrm{~km} \mathrm{~s}^{-1}$ (Zorec et al. 2005), its radius is $6 R_{\odot}$ (Dachs et al. 1989; Prinja 1989) and its mass is $10 M_{\odot}$ (Prinja 1989).

The mass and radius determination of a Be star is not an easy task. For instance if we assume values of masses and radii from the Harmanec (1988) compilation, in agreement with Schaller et al. (1992) non-rotating evolutionary models, for the 
Table 1. Mass and radius determination for $\kappa \mathrm{CMa}$ from the Harmanec (1988) compilation for the effective temperatures given by Popper (1980), Prinja (1989) and Fremat (2005).

\begin{tabular}{cccc}
\hline \hline $\begin{array}{c}T_{\text {eff }} \\
\text { in } \mathrm{K}\end{array}$ & $\begin{array}{c}\text { Mass } \\
\text { in } M_{\odot}\end{array}$ & $\begin{array}{c}\text { Normal radius } \\
\text { in } R_{\odot}\end{array}$ & $\begin{array}{c}\text { Radius from } \\
\text { parallax in } R_{\odot}\end{array}$ \\
\hline 20000 & 6.60 & 3.71 & $7.25(6.46-8.24)$ \\
23100 & 8.62 & 4.28 & $6.26(5.59-7.13)$ \\
25800 & 10.72 & 4.83 & $5.59(4.98-6.36)$ \\
\hline
\end{tabular}

effective temperatures used by Popper (1980), Prinja (1989) and Fremat (2005), we obtain the Table 1.

Thus, for a main sequence star the stellar radius should be smaller than the $6 R_{\odot}$ we have adopted, however, our radius estimate based on the parallax and the chosen $V$ magnitude from the correlation between the brightness and emission strength, as proposed by Harmanec (2000), gives the range of radii comparable to the $6 R_{\odot}$ used in our modeling.

The star exhibits a large IR-excess and strong emission in the hydrogen lines making it a good candidate for the AMBER/VLTI spectro-interferometer (Petrov et al. 2007) using medium spectral resolution (1500). Our aim is to study the geometry and kinematics of the circumstellar environment of this star as a function of wavelength, especially across the Br $\gamma$ emission line and to detect any signatures of a possible asymmetry of its circumstellar disk, as already observed through a violet to red peak ratio $V / R \sim 1.3$ (Dachs et al. 1992; Slettebak et al. 1992).

\section{Observations and data reduction}

Dedicated observations of $\kappa$ CMa were carried out during the night of December 26th 2004 with the three VLTI 8m ESO telescopes UT2, UT3 and UT4 (see Table 3 for the baseline configurations). The data were reduced using the amdlib (v1.15)/ammyorick (v0.54) software package developed by the AMBER consortium. It uses a new data processing algorithm adapted to multiaxial recombination instruments called P2VM (Pixel To Visibility Matrix algorithm). The squared visibility estimator is computed from the basic observable coming from this algorithm, the coherent flux (i.e. complex visibilities frame by frame multiplied by the flux) and the estimated fluxes from each telescope. The principles of the general AMBER data reduction are described in more detail by Millour et al. (2004) and Tatulli et al. (2007).

The complex coherent flux allows one to compute differential phase, i.e. the averaged instantaneous phase substracted from achromatic atmospheric OPD and a wavelength-averaged reference phase. This means that the differential phase is the difference between the phase of the source complex visibility and a mean OPD. This leads to an average differential phase equal to zero on the observed spectral window and the lost of the object's phase slope over the wavelengths. This technique allows one to retrieve partial information about the object's phase and is almost equal to the object's interferometric phase if we have some spectral channels in which we know that the object's phase is zero.

It also allows one to compute "differential" visibility (as defined in Millour et al. 2007), i.e. the instantaneous modulus of the complex visibility divided by the averaged visibility in all the wavelengths excepted the working one. This leads to an average differential visibility equal to 1 in the continuum. It has the advantage over the "classical" visibility estimator of being
Table 2. Calibration star diameters estimated from spectro-photometric indices (computed as in Bonneau et al. 2006) and their associated errors.

\begin{tabular}{ccc}
\hline \hline Star & Diameter (mas) & Error (mas) \\
\hline HD 75063 & 0.50 & 0.08 \\
HD 93030 & 0.454 & 0.006 \\
\hline
\end{tabular}

almost insensitive to rapid frame-to-frame variations of visibility (due to vibrations or atmospheric jittering for example) and therefore one can expect the differential visibility observed to be more precise than the classical visibility estimator given the current vibrations in the VLTI infrastructure, and even though the continuum visibility information is lost in this observable.

Differential data reduction is described in detail in Millour et al. (2007).

Reducing the $\kappa$ CMa data with good accuracy is difficult to achieve. We encountered specific problems related to this data set. Therefore, in addition to the tools furnished by the default package, some specific processing was added to reach the best precision on the interferometric observables.

- No specific data were available to calibrate the fringe contrast of $\kappa$ CMa. We therefore looked at calibration stars observed during the same night for other stars and corrected their visibilities averaged over the [2.13-2.21] $\mu \mathrm{m}$ observed spectral range from their estimated diameters (see Table 2) to monitor the instrumental+atmospheric transfer function (see Fig. 1). This transfer function is the visibility of a point source measured by the instrument, allowing us to correct the raw visibilities for the science star for the instrumentalspecific visibility loss. The scattering over time of the visibilities gives the dispersion due to the instrumental drifts and atmospheric fluctuations during the observing time. This leads to a visibility dispersion estimate of 0.05 for each star, which leads to an error on the calibrated visibilities of $0.07\left(\sqrt{2 \times 0.05^{2}}\right)$.

Then we interpolate the estimated transfer function to the time of the science star observations (as in Perrin et al. 2003). The [2.13-2.21] $\mu \mathrm{m}$ averaged visibility of $\kappa \mathrm{CMa}$ is close to 1.0 with an uncertainty of 0.07 on all the observed base lengths. This would normally be unacceptable for the wavelength-dependence study of the visibilities, but as explained before, we expect to have differential visibility and differential phase estimators that are much more precise than the visibility estimator.

- The lack of dedicated calibration star for $\kappa$ CMa should have lead to an inability to spectrally calibrate the differential observables, but fortunately another calibration star (HD 93030) was observed two hours later within almost the same spectral window, which means that the spectrograph grating did not move but that the detector window was not exactly the same as for $\kappa \mathrm{CMa}$, allowing us to use the intersecting spectral channels between the two observations without any calibration problems. Detailed data analysis of calibration stars tends to demonstrate that the main pattern on differential observables comes from a fiber-injection pattern (i.e. an AMBER internal instrumental effect) and that it is stable over several minutes in the $10^{-2}$ range for the differential visibilities and $10^{-2}$ radians for the differential phase at medium spectral resolution $(R \sim 1500$, see for instance Vannier et al. 2005).

This allowed us to correctly calibrate the differential visibility and the differential phases (see Fig. 2). In order to ensure our 


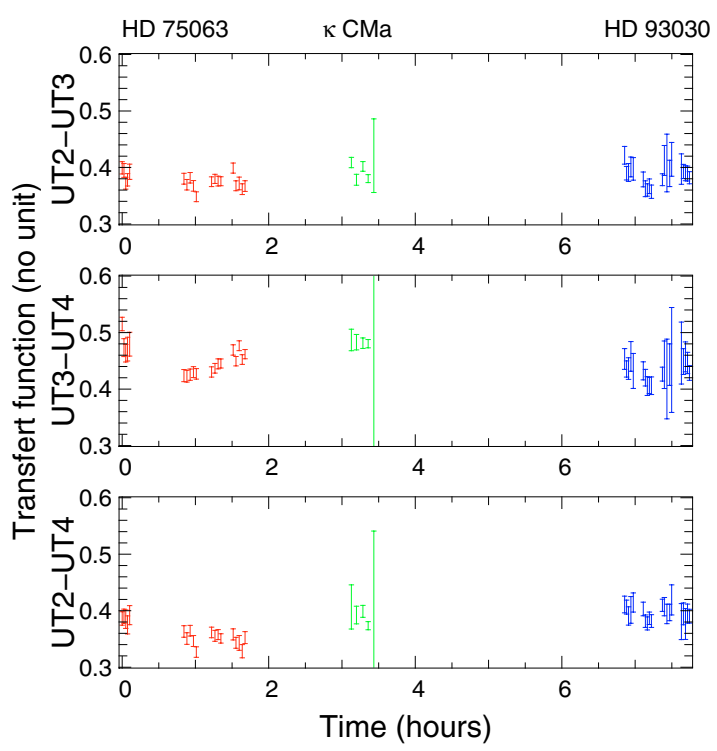

Fig. 1. Raw absolute visibilities of calibration stars corrected for their angular diameters and averaged over the [2.13-2.21] $\mu \mathrm{m}$ window, allowing us to monitor the instrumental+atmospheric transfer function (points respectively around $1 \mathrm{~h}$ in red and $7 \mathrm{~h}$ in blue). For comparison we have overplotted the raw visibilities of $\kappa \mathrm{CMa}$ (around $3 \mathrm{~h}$ in green). The $\kappa \mathrm{CMa}$ visibilities have the same value as the instrumental+atmospheric transfer function within the error bars, leading to a calibrated visibility of 1 , i.e. a non resolved or poorly resolved object on all baselines.

calibration, we checked that all the features mentioned in this article are present in the uncalibrated data, and not added by noise effects produced by the calibrator star.

We could expect to see an effect in the closure phase, but its modulation seems to be of the order of the amplitude of the error bars ( $3^{\circ}$ or 0.05 radians), which means that we do not see any detectable signal in the closure phase. This non-detection confirms the result of the visibility and the low amplitude of the modulation on the differential phases: the object is almost non-resolved or barely resolved by the interferometer on the considered baselines (80 m maximum).

What we see in the observed data is a decrease in the differential visibilities in two of the three baselines of the order of 0.07 , much larger than the error bars $(0.02$ for the differential visibilities). This can be explained by an envelope larger than the star, visible in the emission line.

We observe also a modulation in the differential phase of the order of $5^{\circ}(0.09$ radians $)$, also higher than the error bars $\left(2^{\circ}\right.$ or 0.03 radians). The modulation of the differential phase show a "sine arch" shape, typical of a rotating object or a bipolar outflow but also shows an asymmetry, mainly on the baseline UT2-UT3 (B1).

In order to obtain more kinematical constraints the star has also been observed in the $J 2$ band $(1.2283-1.2937 \mu \mathrm{m})$ with the $1.6 \mathrm{~m}$ Perkin-Elmer telescope and Coudé spectrograph (with $R=10000$ ) at the Observatório do Pico dos Dias, Laboratório Nacional de Astrofísica (LNA), Itajubá, Brasil. The spectra were recorded on the night of 20/21 November 2005, at seven different positions along the slit using the Câmara Infravermelho (CamIV) detector. The images of the darkfield were subtracted from each star's spectral image, wavelength calibration image and five flat-field images. For the sky image we obtained the median combination of the star's spectral images (divided previously by the average of flat-field images). The sky image was
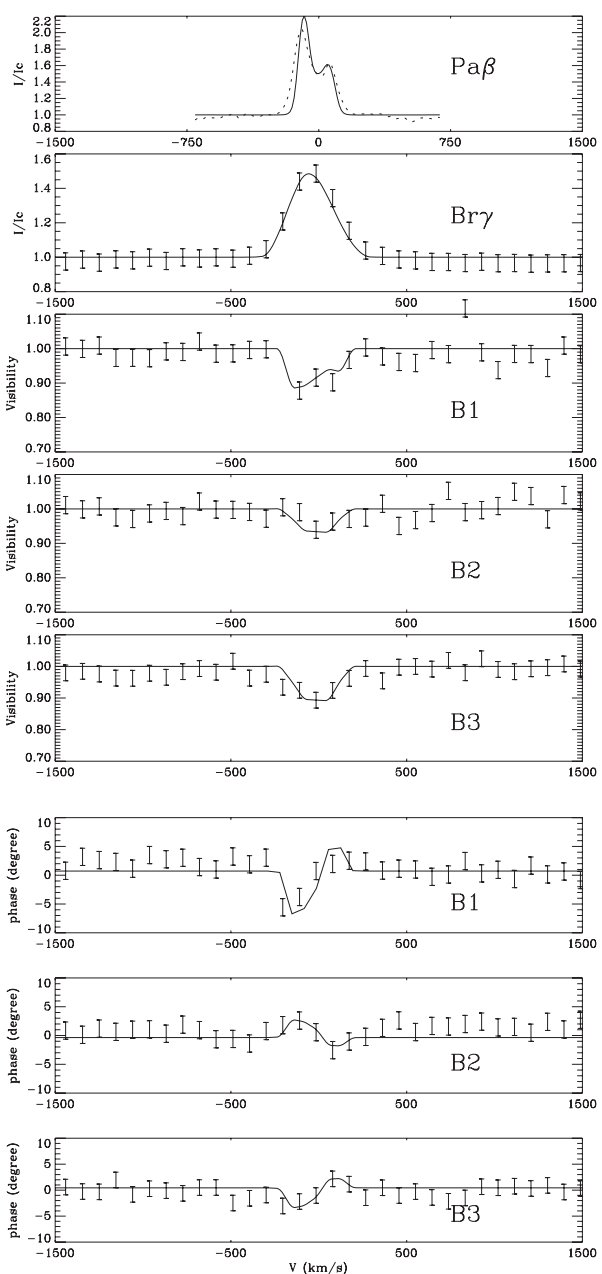

Fig. 2. From top to bottom: $\mathrm{Pa} \beta$ line profile from the Observatorio do Pico dos Dias, Brazil (dotted line) with our best model fit (plain line), Br $\gamma$ line profile, differential visibilities and differential phases for the three baselines. For each plot, the dots with errors bars are AMBER/VLTI data and the solid line is from our best SIMECA model (see Sect. 4).

subtracted from stellar images and the one-dimensional spectra were extracted and calibrated in wavelength using the standard IRAF ${ }^{1}$ procedures. The continuum normalization around the $\mathrm{Pa} \beta$ line was performed using our software. The average profile of the line, which was used to constrain the kinematics within the disc, is plotted in Figs. 2 and 5.

\section{Envelope extension and flattening}

In this section we present the AMBER data to obtain an estimate of $\kappa$ CMa's envelope geometry and extension. Assuming that the measured visibility in the continuum, $V_{\mathrm{c}}$, is only due to the central star and its circumstellar envelope and that the envelope is optically thin in the continuum, we can write:

$V_{\mathrm{c}}=\frac{V_{\mathrm{ec}} F_{\mathrm{ec}}+V_{\star} F_{\star}}{F_{\mathrm{c}}}$

1 IRAF is distributed by the National Optical Astronomy Observatories, which is operated by the Association of Universities for Research in Astronomy (AURA), Inc., under cooperative agreement with the National Science Foundation. 


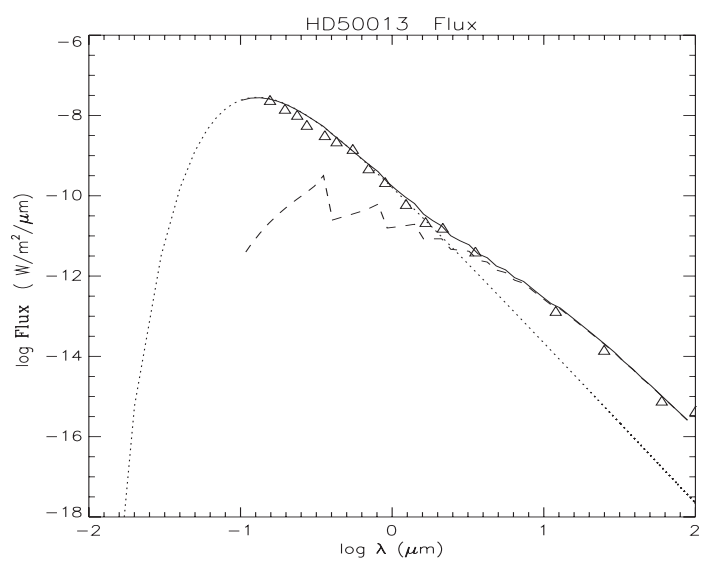

Fig. 3. $\kappa$ CMa's Spectral Energy Distribution (SED) from SIMBAD CDS (triangles). Dotted line: emission from the central star assuming a black body with $R_{\star}=6 R_{\odot}, T_{\text {eff }}=22500 \mathrm{~K}$ and $d=230$ pc. Dashed line: free-free and free-bound envelope contribution from the SIMECA code between 0.3 and $100 \mu \mathrm{m}$. Plain line: Central star emission + envelope contribution.

where $V_{\mathrm{ec}}$ and $F_{\mathrm{ec}}$ are respectively the envelope visibility and flux in the continuum, $V_{\star}$ and $F_{\star}$ are the star visibility and flux in the continuum and $F_{\mathrm{c}}=F_{\text {ec }}+F_{\star}$.

The total flux is normalized, i.e. $F_{\mathrm{c}}=F_{\mathrm{ec}}+F_{\star}=1$. Since the star is fully unresolved $\phi_{\star} \sim 0.25$ mas (assuming a $6 R_{\odot}$ seen at $230 \mathrm{pc}$ ) which corresponds to $V_{\star}>0.99$ for the longest baseline at $2.1 \mu \mathrm{m}$, we assume in the following that $V_{\star}=1$. In order to estimate $V_{\text {ec }}$ we still have to determine the star and the envelope contributions at $2.1 \mu \mathrm{m}$. Using the fit of the SED given in Fig. 3 we estimate that at this wavelength the stellar emission is similar to the envelope contribution, i.e. $F_{\star}=F_{\text {ec }}=0.5$.

We have the same relation for the visibility in the $\operatorname{Br} \gamma$ line:

$V_{\mathrm{r}}=\frac{V_{\mathrm{er}} F_{\mathrm{er}}+V_{\mathrm{c}} F_{\mathrm{c}}}{F_{\mathrm{r}}}$

where $V_{\mathrm{r}}$ and $F_{\mathrm{r}}$ are respectively the measured visibility and flux in the $\mathrm{Br} \gamma$ line. $V_{\mathrm{c}}$ and $F_{\mathrm{c}}$ are previously defined and $V_{\mathrm{er}}$ and $F_{\text {er }}$ are the visibility and flux only due to the $\operatorname{Br} \gamma$ line, i.e. without any stellar contribution and envelope continuum. Using the AMBER Br $\gamma$ emission line profile plotted in Fig. 2 and neglecting the underlying broadened photospheric absorption line, we obtain $F_{\text {er }}=0.5$ and $F_{\mathrm{r}}=1.5$ at the center of the line.

The corresponding visibilities, deduced from Eqs. (1) and (2) and from the measurements shown in Fig. 2, are given in Table 3. Using a uniform disk model for the envelope contribution, for each measurement, we also estimate in Table 3 the corresponding angular diameters in the continuum and in the $\mathrm{Br} \gamma$ line. Since the envelope is marginally resolved in the continuum we simply put an upper limit to its angular size.

The envelope extensions in $\mathrm{Br} \gamma$ given in Table 3 are strongly dependent on the sky-plane baseline orientation as seen in Fig. 4, where we plotted the $\kappa$ CMa (unresolved star + uniform disk) model diameters as a function of the baseline orientation.

The $\kappa$ CMa circumstellar disk seems to be elongated along $B_{1}$ but since we only have 3 visibility measurements we cannot accurately determine the angular position of the majoraxis assuming an elliptical circumstellar disk. The envelope flattening given by the semi-major and semi-minor axis ratio, is about $2 \pm 0.7$. Assuming that the disk is geometrically thin (i.e. its opening angle is only a few degree) we can estimate the range for the inclination angle $i: 39^{\circ}<i<68^{\circ}$. The lower limit of $39^{\circ}$ relies on the lack of constraint on the disk opening angle.
Table 3. $\mathrm{Br} \gamma$ visibilities measured in the continuum $\left(V_{\mathrm{c}}\right)$ and visibility drop within the $\mathrm{Br} \gamma$ line $\left(V_{\mathrm{r}} / V_{\mathrm{c}}\right) . V_{\mathrm{r}}$ calculated from the measured $V_{\mathrm{c}}$ and $V_{\mathrm{r}} / V_{\mathrm{c}}$ ratio. The deduced envelope contribution in the continuum $\left(V_{\mathrm{ec}}\right)$ and in the line $\left(V_{\text {er }}\right)$ is given for each baseline. The corresponding angular diameters in the $\operatorname{Br} \gamma$ line $\left(\phi_{\mathrm{er}}\right)$ and the nearby continuum $\left(\phi_{\mathrm{ec}}\right)$ are computed using a uniform disk model for each envelope measurement. The corresponding extension in stellar radii are also given, assuming a $6 R_{\odot}$ star at $230 \mathrm{pc}$.

\begin{tabular}{c|ccc}
\hline \hline Base $\mathrm{n}^{\circ}$ & 1 & 2 & 3 \\
\hline Baseline & $\mathrm{UT} 2-3$ & $\mathrm{UT3}-4$ & $\mathrm{UT} 2-4$ \\
Length $(\mathrm{m})$ & 42.7 & 59.3 & 80.8 \\
$\mathrm{PA}\left({ }^{\circ}\right)$ & 51.6 & 128 & 97.1 \\
\hline$V_{\mathrm{c}}$ & $>0.93$ & $>0.93$ & $>0.93$ \\
$V_{\mathrm{r}} / V_{\mathrm{c}}$ & $0.93 \pm 0.02$ & $0.95 \pm 0.02$ & $0.9 \pm 0.02$ \\
$V_{\mathrm{r}}$ & $0.85<V_{\mathrm{r}}<0.95$ & $0.87<V_{\mathrm{r}}<0.97$ & $0.82<V_{\mathrm{r}}<0.92$ \\
\hline$V_{\mathrm{ec}}$ & $>0.86$ & $>0.86$ & $>0.86$ \\
$V_{\mathrm{er}}$ & $0.69<V_{\mathrm{er}}<0.85$ & $0.75<V_{\mathrm{er}}<0.91$ & $0.60<V_{\mathrm{er}}<0.76$ \\
\hline$\phi_{\mathrm{ec}}(\mathrm{mas})$ & $<3.6$ & $<2.6$ & $<1.9$ \\
$\phi_{\mathrm{er}}(\mathrm{mas})$ & $3.7<\phi_{\mathrm{er}}<5.5$ & $2.0<\phi_{\mathrm{er}}<3.6$ & $2.6<\phi_{\mathrm{er}}<3.4$ \\
\hline$\phi_{\mathrm{ec}}\left(\mathrm{D}_{\star}\right)$ & $<15.5$ & $<11.2$ & $<8.2$ \\
$\phi_{\mathrm{er}}\left(\mathrm{D}_{\star}\right)$ & $15.9<\phi_{\mathrm{er}}<23.7$ & $8.6<\phi_{\mathrm{er}}<15.4$ & $11.2<\phi_{\mathrm{er}}<14.6$ \\
\hline \multicolumn{4}{r}{}
\end{tabular}

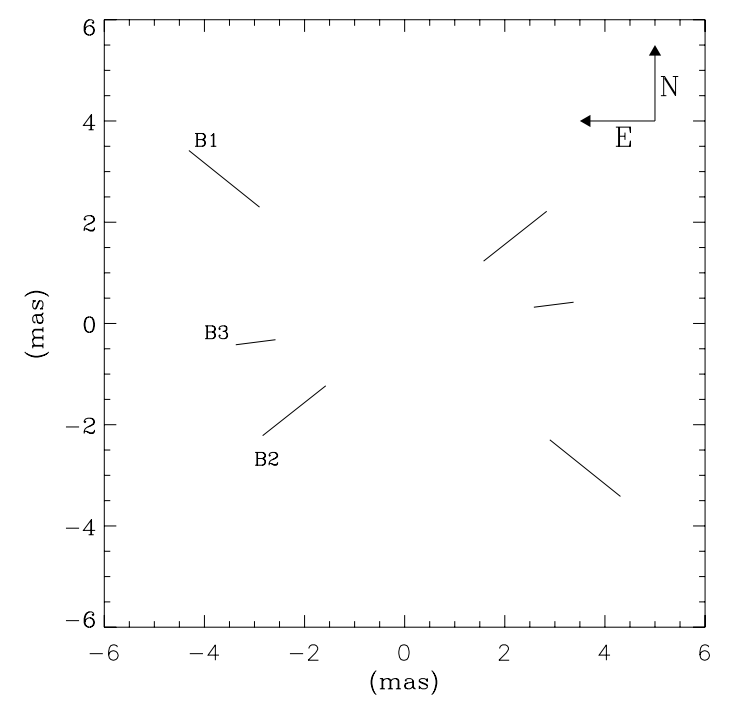

Fig. 4. $\kappa \mathrm{CMa}$ diameters in the $\mathrm{Br} \gamma$ line, assuming an unresolved star + uniform disk models, as a function of the baseline position angle (in mas). The length of each plot corresponds to the error bar measurement whereas diameters are given by the center of each error bar.

\section{SIMECA modeling}

In order to obtain quantitative fundamental parameters of the central star and its circumstellar disk, we used the SIMECA code developed by Stee (1994) and Stee \& Bittar (2001) to model the $\kappa$ CMa circumstellar environment. Since this code was axi-symmetric we made substantial modifications in order to introduce a longitudinal dependence of the envelope density as shown in the AMBER data plotted Fig. 2. To constrain the kinematics within the disk we use a $\mathrm{Pa} \beta$ line profile obtained in December 2005 at the Observatorio do Pico do Dios, Brazil and plotted in Fig. 5. This profile is strongly asymmetric with a $V / R$ double peak of $\sim 1.3$. This $V / R>1$ is usually interpreted in terms of a viscous disk similar to accretion disks where the gas and angular momentum are diffused outward by magnetohydrodynamic viscosity (Lee et al. 1991). Considering the time-dependent structure of the isothermal viscous disk, Okazaki (1997) showed that "one-armed" density waves can propagate within the disk and should reproduce the observed 




Fig. 5. Upper picture: $\kappa \mathrm{CMa} \mathrm{Pa} \beta$ line profile observed in December 2005 at the Observatorio do Picos dos Dias, Brazil (solid line). Estimated symmetric part of the $\mathrm{Pa} \beta$ profile (dotted line) using an axi-symmetric model. The asymmetric residue corresponds to the emission of "one-armed" over-density (dashed line). Bottom picture: differential phase variation measured along the $\mathrm{B}_{1}$ baseline (dots with errors bars) and theoretical phase from the SIMECA code.

$V / R$ variations from $V / R>1$ to $V / R<1$ seen in the line profiles (Hummel \& Hanuschik 1997). Such variations were detected for many Be stars, with periods from a few years to over a decade (Hanuschik et al. 1995; Telting et al. 1994). But in the case of $\kappa$ CMa the $V / R$ ratio has remained constant for the last twenty years (Dachs et al. 1992; Slettebak 1992).

In Fig. 5 we over-plotted the supposed "symmetric part" of the $\mathrm{Pa} \beta$ line profile, using an axi-symmetric model, and the asymmetric residual that may be produced within the "onearmed" oscillation over-density. This effect must be compatible with the asymmetric differential phase variation across the $\mathrm{Br} \gamma$ line for the $\mathrm{B}_{1}$ baseline plotted in the bottom part of Fig. 5 since the emitting regions in $\mathrm{Pa} \beta$ and $\mathrm{Br} \gamma$ must be very close each together. The asymmetric contribution to the $\mathrm{Br} \gamma$ emission is about 20 to $30 \%$ of the total emission in this line whereas the mean projected velocity of the inhomogeneity is $-130 \pm 20 \mathrm{~km} \mathrm{~s}^{-1}$. Using a SIMECA model at $230 \mathrm{pc}$ we determined that the projected separation between this over-density photocenter and the central star is about $6.5_{\star}$.

The parameters obtained for our best model are given in Table 4 with the corresponding spectroscopic and interferometric observables of Fig. 2. This best model includes an overdensity along the disk major axis at $+20^{\circ}$, corresponding to an over-luminosity of $30 \%$ of the total flux in the line, and the agreement with the AMBER/VLTI data, the SED (Fig. 3) and the $\mathrm{Pa} \beta$ line profile is very good, as can be seen in Fig. 2 . The agreement with the differential visibility and phase across the $\mathrm{Br} \gamma$ line for the three bases validates the chosen disk geometry and kinematics. The $2.1 \mu \mathrm{m}$ continuum visibilities obtained with the 3 baselines, $V_{1}=0.92, V_{2}=0.96$ and $V_{3}=0.94$ are also compatible with the 0.93 lower limit measured with AMBER. The corresponding continuum intensity map in the continuum at $2.15 \mu \mathrm{m}$ is plotted in Fig. 6. The evaluation of the uncertainties of the parameters of our model is beyonf the scope of this work and will be studied in depht when more constraining data is available.
Table 4. Parameters for the $\kappa$ CMa central star and its circumstellar environment for the best axi-symetric model.

\begin{tabular}{cc}
\hline \hline parameter & value \\
\hline$T_{\text {eff }}$ & $22500 \mathrm{~K} \pm 1000$ \\
Radius & $6 R_{\odot} \pm 1$ \\
Inclination angle $i$ & $60^{\circ} \pm 10$ \\
Equatorial rotation velocity & $240 \mathrm{~km} \mathrm{~s}^{-1} \pm 20$ \\
rotation law exponent & $0.32 \pm 0.1$ \\
Photospheric density $\left(\rho_{\text {phot }}\right)$ & $4 \times 10^{-11} \mathrm{~g} \mathrm{~cm}^{-3} \pm 2 \times 10^{-11}$ \\
Equatorial terminal velocity & $1 \mathrm{~km} \mathrm{~s}^{-1} \pm 10$ \\
Polar terminal velocity & $1000 \mathrm{~km} \mathrm{~s}^{-1} \pm 100$ \\
Polar mass flux & $M_{\odot} \mathrm{year}^{-1} \mathrm{sr}^{-1} \pm 0.5 \times 10^{-11}$ \\
m1 & $10 \pm 5$ \\
m2 & $10 \pm 2$ \\
C1 & $30 \pm 10$ \\
Envelope outer radius & $23 R_{\star} \pm 2$ \\
Major axis position & $+28^{\circ} \pm 5$ \\
Over-density position & along the disk major axis \\
\hline
\end{tabular}

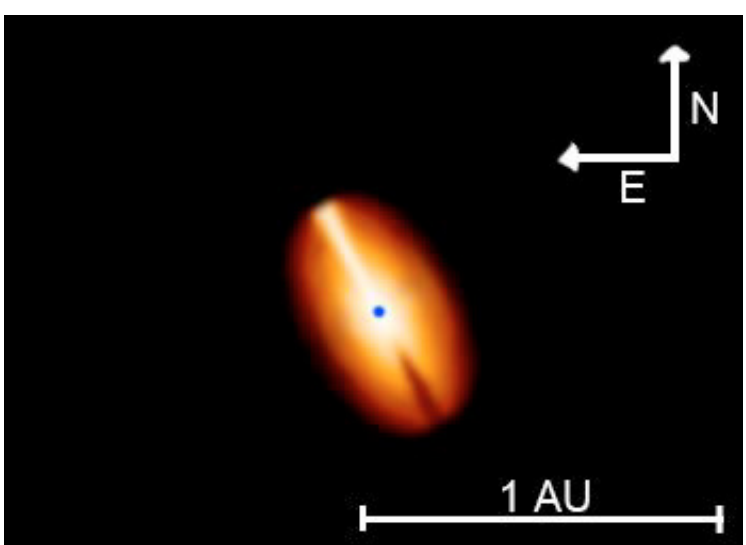

Fig. 6. Intensity map in the continuum at $2.15 \mu \mathrm{m}$ obtained with SIMECA for our best model parameters. The inclination angle is $60^{\circ}$, the central black dot represents the $\kappa$ CMa photosphere ( 0.25 mas); the bright part in the equatorial disk is produced by the over-density which is oriented along the $\mathrm{B}_{1}$ baseline. This over-density is also responsible for a $30 \%$ emission excess in the asymmetric $V$ part of the Bry line.

\section{Discussion}

Following recent AMBER/VLTI and MIDI/VLTI observations of $\alpha$ Arae, Meilland et al. (2007) concluded that this classical Be star fits very well within the classical scenario for the "Be phenomenon", i.e. a fast rotating B star close to its breakup velocity surrounded by a Keplerian circumstellar disk with an enhanced polar wind. This scenario was also confirmed for the Be star Achernar by Kervella \& Domiciano de Souza (2006) using VLTI/VINCI data, even if, for this latter case, the star was not in its active Be phase, i.e. without any strong emission line and no circumstellar disk. Nevertheless, Achernar is still a nearly critical rotator and shows an enhanced polar stellar wind. We will see in the following that $\kappa$ CMa does not fit very well within this classical scenario.

\subsection{Is к CMa a critical rotator?}

If $\kappa \mathrm{CMa}$ was rotating close to its breakup velocity, i.e. $V_{\mathrm{c}}=$ $463 \mathrm{~km} \mathrm{~s}^{-1}$, the inclination angle would be around $28^{\circ}$ in order to obtain a measured $v \sin i=220 \mathrm{~km} \mathrm{~s}^{-1}$. With this inclination angle the maximum flattening corresponding to a geometrically very thin disk is 1.12 . Since we measure a flattening of 
about $2 \pm 0.7$, this inclination angle can be ruled out. In our best SIMECA model the star is rotating at only $52 \%$ of its critical velocity. We may argue that the measured elongation is not the envelope major axis but rather the enhanced polar wind. In this case the projected axis of the Be envelope is not identical to the rotation axis of the star. Nevertheless, in order to obtain an asymmetry in the jet we need an extended optically thick disk, perpendicular to the jets directions, that may screen at least one part of the jet-like structure. Such an extended optically thick disk should have been detected with the AMBER instrument which is not the case in our data.

The value of the projected rotational velocity for an early-B star can be systematically affected by pseudo-photosphere, unrecognized optically thick parts of the Be envelope as shown by Harmanec (2002) for $\gamma$ Cas. He obtains for this star a $v \sin i$ of $380 \mathrm{~km} \mathrm{~s}^{-1}$ instead of the often quoted value of $230 \mathrm{~km} \mathrm{~s}^{-1}$ from Sletteback (1992). Nevertheless, taking the largest value for $\kappa \mathrm{CMa}$ from the literature from Zorec (2005) who found a $v \sin i=243 \mathrm{~km} \mathrm{~s}^{-1}$ we still obtain an inclination angle of $32^{\circ}$ which again is not in agreement with our measured flattening. If the discrepancy between the measured $v \sin i$ and the "real" one is larger it may be possible that $\kappa \mathrm{CMa}$ is still a critical rotator but it requires a factor of 2 between the measured and the true $v \sin i$, which we found unrealistic. Even if Townsend et al. (2004) include the gravity darkening effect on the $v \sin i$ values of rigid early-type rotators, assuming a rotation rate $\Omega / \Omega_{\mathrm{c}}$ of 0.95 , they conclude that classic $v \sin i$ determinations for B0 to B9-type stars can be underestimated by 12 to $33 \%$, far from a factor of 2. Moreover, a recent paper by Frémat et al. (2005) studying the effect of the gravitational darkening on the determination of fundamental parameters in fast rotating B-type stars found that on average the rate of angular velocity of Be stars attains only $\Omega / \Omega_{\mathrm{c}} \sim 0.88$.

Frémat et al. (2005) estimate $\kappa$ CMa's effective temperature to be $25790 \pm 713 \mathrm{~K}$, a value significantly larger than the $22500 \mathrm{~K}$ used in our modeling. Moreover, Harmanec (2000) found a positive correlation between the emission strength and brightness in the optical. Therefore we may use the minimum observed $V$ magnitude of about 3.5 to estimate the radius of the central star. Combining with the Hipparcos parallax and its error we obtain a radius between 9 and 14 solar radii. Using the $T_{\text {eff }}$ of $25790 \mathrm{~K}$ and a radius of $14 R_{\odot}$ we obtain a stellar luminosity larger by a factor of 8 than our modeling and thus it is not possible to obtain a good agreement with the observed SED plotted Fig. 3. We are more confident in our $6 R_{\odot}$ used for our modeling and our finding that $\kappa \mathrm{CMa}$ seems not to be a critical rotator. Nevertheless, regarding the uncertainties and the large errors of all measurements the breakup velocity cannot be totally excluded.

\subsection{Is the rotation law within the disk Keplerian?}

A Keplerian rotation law would produce a narrower doublepeaked separation in the $\mathrm{Pa} \beta$ line profile. Using a simple axisymmetric Keplerian disk model the double-peak separation would be about $90 \mathrm{~km} \mathrm{~s}^{-1}$ whereas we measure an asymmetric double-peak separation of about $160 \mathrm{~km} \mathrm{~s}^{-1}$. Even if we subtract the emission of the over-density producing a larger double-peak separation by contributing to the $V$ peak of the emitting $\mathrm{Pa} \beta$ line, we still obtain a double-peak separation of about $120 \mathrm{~km} \mathrm{~s}^{-1}$ (see Fig. 5). The exponent of the rotation law used for our best SIMECA model is 0.32 whereas it should be 0.5 for a purely Keplerian disk.
We may argue that Be stars vary strongly in time and thus line profile shapes are time dependent. For instance, actual $\mathrm{H} \alpha$ line profiles show a strong emission with a single peak whereas Bahng \& Hendry (1975) saw a double-peaked H $\alpha$ emission line, with the same double-peak separation of $160 \mathrm{~km}^{-1}$ we obtained for $\mathrm{Pa} \beta$ with a shell core in their high-dispersion spectra. Nevertheless, these line variations are related to the formation and disappearance of the circumstellar disk around the star as shown by Rivinius et al. (2001) and Meilland et al. (2006). Whatever the model is, a double-peak line profile is a clear signature of an extended rotating disk, at least if the kinematics are not dominated by a strong stellar wind in the equatorial region as shown by Stee \& de Araùjo (1994). This double-peaked separation is a good indication of the disk extension as shown by Huang (1972), Hirata \& Kogure (1984), and Stee \& de Araùjo (1994). We measure $v_{\text {disk }} \sin i$ at the disk outer radius $\left(R_{\text {disk }}\right)$ from the peak separation, where $v_{\text {env }}$ is the rotational disk velocity at $R_{\text {disk }}$. Thus we can write:

$v_{\text {disk }} \sin (i)=v_{\star} \sin (i)\left(\frac{R_{\text {disk }}}{R_{\star}}\right)^{-\beta}$,

where $v_{\star}$ is the star rotation at its photosphere.

Assuming a Keplerian rotation $(\beta=0.5)$ we obtain, using Eq. (3), $R_{\text {disk }}=13.5 R_{\star}$ which is about $2 \sigma$ from the $19.8 R_{\star}$ interferometric measurement, assuming that the measured elongation is the envelope major axis and not an enhanced polar wind (see discussion in the previous point). Note that these $19.8 R_{\star}$ found are obtained assuming a uniform disk for the envelope and thus is certainly a lower limit to the "true" disk extension in the $\mathrm{Pa} \beta$ line. Thus it seems difficult to maintain a Keplerian rotation within the disk of $\kappa \mathrm{CMa}$.

\subsection{Is the "one-armed" viscous disk model a possible scenario for $\mathrm{K} C \mathrm{CM}$ ?}

The asymmetry presently detected in the disk of $\kappa$ CMa seems to be poorly explained within the "one-armed" viscous disk framework. Following the viscous disk models by Okazaki (1997) and the observational detection of "one-armed" oscillations in the disk of $\zeta$ Tau by Vakili et al. (1998) and $\gamma$ Cas by Berio et al. (1999), the precessing period (P) of such oscillations should be confined within a few years up to about twenty years for the longer ones. We tried to compile all the observational data available to obtain a "quasi-period" for the $V / R$ variations. The $V / R$ variations occur during the time intervals of observable presence of Be envelopes and that they can show longterm, medium-term as well as rapid changes (Dachs 1981). Moreover, the very strong $\mathrm{H} \alpha$ line profile is not suitable for $V / R$ measurement since it is single-peaked and the illusion of apparent $V / R$ changes can be related to the presence of telluric lines. Compiling the data between 1965 and 2003 for $\kappa \mathrm{CMa}$ from Jaschek (1965), Slettebak (1982), Banerjee (2000) and this work, we were not able to deduce an estimation of a quasiperiod (Fig. 7). Several authors suggested a very long $V / R$ variation (i.e. Okazaki $P>28$ years). An equally plausible possibility is that the star had two episodes of $V / R$ changes with much shorter cycle length separated by a period of quiescence documented by (Dachs et al. 1992; Slettebak 1992). More observations are needed since, if this first possiblity could be confirmed, this conflicts with the one-armed model. This "pseudoperiod" would be too long compared to theoretical predictions which cannot be longer than two decades for a disk with a radius $\sim 23 R_{\star}$ (Okazaki, private communication). The fact that 


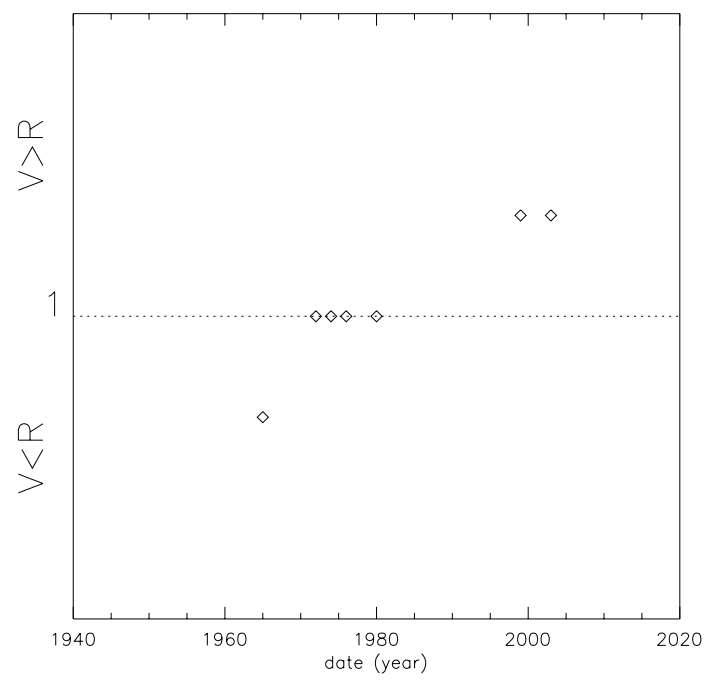

Fig. 7. $V / R$ variations obtained from the literature between 1965 and 2003, from Jaschek (1965), Slettebak (1982), Banerjee (2000) and this work.

this over-density remains confined along the major axis of the disk seems to be only fortuitous...

More observations are needed to confirm these conclusions and to determine whether other physical phenomena occurred within the circumstellar disk of $\kappa \mathrm{CMa}$.

Acknowledgements. We thank A. Okasaki for his useful comments about the viscous disk models. We acknowledge the remarks of the referee P. Harmanec which helped to improve the paper. We thank D. Chapeau and D. Mattei for the SIMECA code developments support.

The AMBER project ${ }^{2}$ was founded by the French Centre National de la Recherche Scientifique (CNRS), the Max Planck Institute für Radioastronomie (MPIfR) in Bonn, the Osservatorio Astrofisico di Arcetri (OAA) in Firenze, the French Region "Provence Alpes Côte D'Azur" and the European Southern Observatory (ESO). The CNRS funding has been made through the Institut National des Sciences de l'Univers (INSU) and its Programmes Nationaux (ASHRA, PNPS, PNP).

The OAA co-authors acknowledge partial support from MIUR grants to the Arcetri Observatory: A LBT interferometric arm, and analysis of VLTI interferometric data and From Stars to Planets: accretion, disk evolution and planet formation and from INAF grants to the Arcetri Observatory Stellar and Extragalactic Astrophysics with Optical Interferometry. C. Gil work was supported in part by the Fundação para a Ciência e a Tecnologia through project POCTI/CTE-AST/55691/2004 from POCTI, with funds from the European program FEDER.

The preparation and interpretation of AMBER observations benefit from the tools developed by the Jean-Marie Mariotti Center for optical interferometry $\mathrm{JMMC}^{3}$ and from the databases of the Centre de Données Stellaires (CDS) and of the Smithsonian/NASA Astrophysics Data System (ADS).

The data reduction software amdlib is freely available on the AMBER site http://amber.obs.ujf-grenoble.fr. It has been linked to the public domain software Yorick ${ }^{4}$ to provide the user-friendly interface ammyorick.

\section{References}

Bahng, J. D. R., \& Hendry, E. 1975, PASP, 87, 137

Banerjee, D. P. K., Rawat, S. D., \& Janardhan, P. 2000, A\&AS, 147, 229

Berio, Ph., Stee, Ph., Vakili, F., et al. 1999, A\&A, 345, 203

Bonneau, D., Clausse, J., Delfosse, X., et al. 2006, A\&A, 4469

Dachs, J., Eichendorf, W., Schleicher, H., et al. 1981, A\&AS, 43, 427

Dachs, J., Poetzel, R., \& Kaiser D. 1989, A\&AS, 78, 487

Dachs, J., Hummel, W., \& Hanuschik, R. W. 1992, A\&AS, 95, 437

2 The structure and members of the AMBER Consortium can be found in the website: http://amber.obs.ujf-grenoble. fr

3 The JMMC is a center providing software tools for optical interferometry described at the website: http://www. jmmc. fr

4 http://yorick. sourceforge.net
Domiciano de Souza, A., Kervella, P., Jankov, S., et al. 2003, A\&A, 407, L47 Frémat, Y., Zorec, J., Hubert, A.-M., et al. 2005, A\&A, 440, 305 Hanuschik, R. W., Hummel, W., Dietle, O., et al. 1995, A\&A, 300, 163 Harmanec, P. 1988, BAICz, 39, 329

Harmanec, P. 2000, Alicante IAU Col. 175, ASP, 214, 13

Harmanec, P. 2002, ASPC, 279, 221

Hirata, R., \& Kogure, T. 1984, Bull. Astr. Soc. India, 12, 109

Huang, S. 1972, ApJ, 171, 549

Hummel, W., \& Hanuschik, R. W. 1997, A\&A, 320, 852

Jaschek, C., \& Jaschek, M. 1965, PASP, 77, 376

Kervella, P., \& Domiciano de Souza, A. 2006, A\&A, 453, 1059

Lee, U., Osaki, Y., \& Saio, H. 1991, MNRAS, 250, 432

Marlborough, J. M., \& Peters, G. J. 1986, ApJS, 62, 875

Meilland, A., Stee, Zorec, J., et al. 2006, A\&A, 455, 953

Meilland, A., Stee, P., Vannier, M., et al. 2007, A\&A, 464, 59

Mennickent, R. E., Vogt, N., \& Barrera, L. H. 1994, A\&AS, 106, 427

Millour, F., Tatulli, E., Chelli, A., et al. 2004, SPIE, 5491, 1222

Millour, F., Petrov, R. G., Chesneau, O., et al. 2007, A\&A, 464, 107

Okazaki, A. 1997, A\&A, 318, 548

Petrov, R. G., Malbet, F., Weigelt, G., et al. 2007, A\&A, 464, 1

Perrin, G. 2003, A\&A, 400, 1173

Popper, D. M. 1980, ARA\&A, 18, 115

Prinja, R. K. 1989, MNRAS, 241, 721

Rivinius, Th., Baade, D., Stefl, S., et al. 2001, A\&A, 379, 257

Schaller, G., Schaerer, D., Meynet, G., et al. 1992, A\&AS, 96, 269

Slettebak, A. 1982, ApJ, 50, 55

Slettebak, A., Collin, G., et al. 1992, ApJS, 81, 335

Stee, Ph., \& de Araùjo, F. X. 1994, A\&A, 292, 221

Stee, Ph., de Araùjo, F. X., Vakili, F., et al. 1995, A\&A, 300, 219

Stee, Ph., \& Bittar, J. 2001, A\&A, 367, 532

Tatulli, E., Millour, F., Chelli, A., et al. 2007, A\&A, 464, 29

Telting, J. H., Heemskerk, M. H. M., Henrichs, H. F., et al. 1994, A\&A, 288, 558 Townsend, R. H. D., Owocki, S. P., \& Howarth, I. D. 2004, MNRAS, 350, 189

Vannier, M., Millour, F., \& Petrov, R. G. 2005, in The power of optical/IR interferometry, ESO Conf., in press

Vakili, F., Mourard, D., Stee, Ph., et al. 1998, A\&A, 335, 261

Zorec, J., Frémat, Y., \& Cidale, L. 2005, A\&A, 441, 235

1 Laboratoire Gemini, UMR 6203 Observatoire de la Côte d'Azur/CNRS, BP 4229, 06304 Nice Cedex 4, France

2 Laboratoire Universitaire d'Astrophysique de Nice, UMR 6525 Université de Nice - Sophia Antipolis/CNRS, Parc Valrose, 06108 Nice Cedex 2, France

3 Laboratoire d'Astrophysique de Grenoble, UMR 5571 Université Joseph Fourier/CNRS, BP 53, 38041 Grenoble Cedex 9, France

4 Max-Planck-Institut für Radioastronomie, Auf dem Hügel 69, 53121 Bonn, Germany

5 INAF-Osservatorio Astrofisico di Arcetri, Istituto Nazionale di Astrofisica, Largo E. Fermi 5, 50125 Firenze, Italy

6 European Southern Observatory, Casilla 19001, Santiago 19, Chile

7 ONERA/DOTA, 29 av de la Division Leclerc, BP 72, 92322 Chatillon Cedex, France

8 Centre de Recherche Astronomique de Lyon, UMR 5574 Université Claude Bernard/CNRS, 9 avenue Charles André, 69561 Saint Genis Laval Cedex, France

9 Division Technique INSU/CNRS UPS 855, 1 place Aristide Briand, 92195 Meudon Cedex, France

10 IRCOM, UMR 6615 Université de Limoges/CNRS, 123 avenue Albert Thomas, 87060 Limoges Cedex, France

11 European Southern Observatory, Karl Schwarzschild Strasse 2, 85748 Garching, Germany

12 Kiepenheuer Institut für Sonnenphysik, Schöneckstr. 6, 79104 Freiburg, Germany

13 Instituut voor Sterrenkunde, KU-Leuven, Celestijnenlaan 200D, 3001 Leuven, Belgium

14 Centro de Astrofísica da Universidade do Porto, Rua das Estrelas, 4150-762 Porto, Portugal

15 Laboratoire Astrophysique de Toulouse, UMR 5572 Université Paul Sabatier/CNRS, BP 826, 65008 Tarbes Cedex, France

16 Departamento de Astronomia, Universidad de Chile, Chile 